\title{
A REPORT OF THIRTEEN CASES OF ATAXIA IN ADULTS WITH HEREDITARY HISTORY.
}

\author{
BY DR. IRWIN H. NEFF, \\ Assistant Physician, Michigan Asylum for the Insane, Kalamazoo, Mich.
}

Friedreich, in a description of the disease which bears his name, emphasized certain characteristic symptoms, and, on the basis of his description, adversely criticised many cases which were then presented as examples of this form of ataxia. Ladame (Brain: Winter Number, 1890), in his historical and critical digest of the malady, strongly supports and corroborates the original description of the disease. The following summary of the diagnostic points is taken from his article:

"Slow and progressive weakness and ataxia of the legs, developing gradually in childhood, almost always without pains, but often simulating choreiform movements. Very slow progressive course from below upward, which attacks successfully the trunk, the arms, the muscles of the larynx, of the tongue and of the eyes. Ataxic and trembling gait, becoming gradually aggravated. No remissions. Speech slow, drawling, scanned, as in disseminated sclerosis, static and dynamic nystagmus; abolition of knee reflexes; scoliosis; peculiar deformity of the foot."

To this he adds the following negative symptoms:

"Absence of lightning pains, integrity of cutaneous sensations in all its manifestations. Normal reaction of pupils, integrity of sight. Absence of genito-urinary troubles. No syphilitic antecedents. Family character of the disease."

Sanger Brown (Brain: Part 2, 1892) gives an interesting review of the disease. Twenty-five cases occurred in one family which he reported, but these differed in essential particulars from the clinical type of the malady. The author acknowledged the difference of symptomatology, but thinks that, notwithstanding the absence of characteristic symptoms and the apparent contradiction, they should be classified as examples of the disease, or should denote an unrecognized form of hereditary ataxia. But few of the cases which have been reported have corresponded to the form described by the discoverer. The cases reported below are similar in some respects to those reported by Sanger Brown, but have peculiarities as regards certain symptoms. 
Recently cases have been reported without hereditary history. This adds to the confusion and renders difficult a recognition of the trouble as originally described, and in many cases the types reported differ in essential particulars from the original report of Friedrich.

Before giving the results of the examination of the two cases which I have examined, I think it best to give the history of other members of the family similarly afflicted, in the order of advancement.

Reference to the accompanying chart will enable the reader to easily follow the description.

Case 1.- Great-grandfather on the paternal side was said to have "paralysis." His age at the commencement of the disease was sixty. He gradually lost power in speaking, dying at an advanced age; cause unknown.

Case 2.- The paternal grandmother developed the disease at the age of sixty-two. She was at first unable to walk, then had difficulty in moving her arms, and trouble in speaking developed in a short time. She became entirely helpless several years before death, and is supposed to have died from the effects of the disease.

Case 3.- Father of patient. First sympt'sm noticed at the age of fifty-five. This was impaired utterance, and in a few months inability to stand. It is thought by the relatives that the latter symptom was present before the disorder of speech was declared, but no positive information can be gained concerning this. In the course of the disease there was pronounced difficulty in moving the hands, and during the last seven years of his life he was unable to take a step without assistance. Died at the age of sixty-seven; cause unknown.

Case 4.- Lewis, brother of the above. Developed the disease at the age of sixty. Symptoms and the course of the malady the same as the preceding case. Difficulty in walking, incorordination of muscles of arms and disturbance of speech. Age and cause of death unknown. For three inonths before death he "lost his mind." (From description, I should judge it to be a form of dementia.)

Case 5.- Jacob, brother of the above, was attacked at the age of sixty. The onset and progress of disease was the same as last case related. He was unable to walk alone, and became helpless eight years before decease. Died at the age of seventy-two. Death supposed to be directly due to asthenia from effects of the disease. 
1885.]

BY IRWIN H. NEFF, M. D.

867

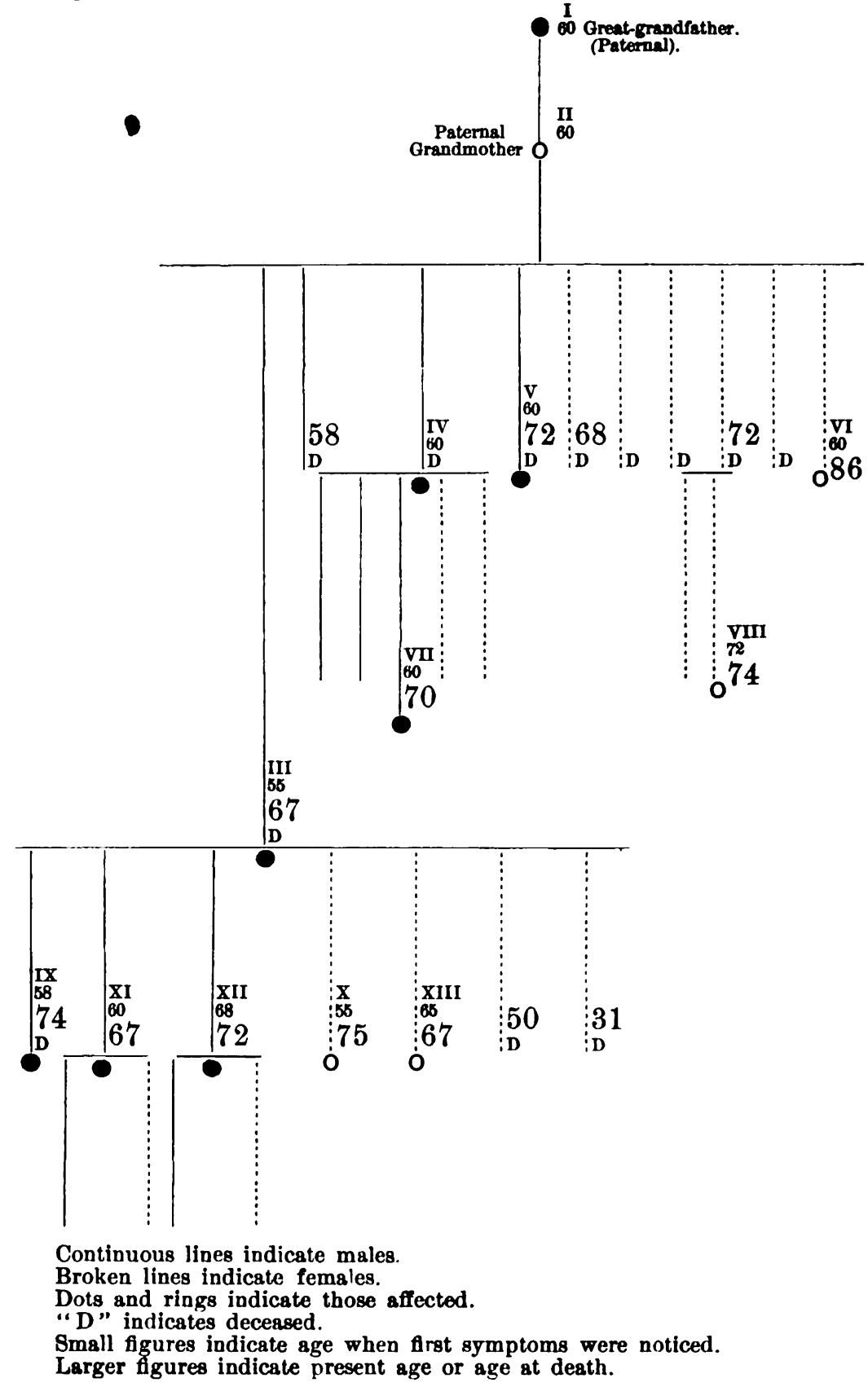


Case 6. - Emily, sister of the above. Is the only one living of the third generation. The first symptoms were noticed at the age of sixty, and its progression from lower to upper extremities and involvement of the speech was coincident with the others afflicted. She is now aged eighty-six and, although unable to walk alone, is remarkably well preserved for her age. Mind is unaffected.

Case 7.- Norman, son of Lewis. Developed the malady at the age of sixty. Primarily had difficulty in locomotion. Now, at the age of seventy, has difficulty in moving his arms, "can not speak plainly," but is able to move around, although he is quite unsteady.

Case 8.-Mrs. W., daughter of Sylvia, age seventy-four, developed the disease at the age of seventy-two. The oldest of the reported cases, of whom I have any knowledge, developing the trouble at this advanced age. In her case the course has been insidious, and at the present time she is quite "shaky," and the trouble is making slow and steady progress.

Case 9.- Brother of patient. Was afflicted with the disease at the age of fifty-eight. First symptom was inability to stand, speech was affected one year after onset. He was able to walk until one year before his death, which occurred at the age of seventy-four. Supposed cause of death, exhaustion from the disease.

Case 11.- Brother of the above. Age sixty-seven. Has the disease in an aggravated form. First symptom noticed at the age of sixty. Had difficulty in uttering some words, and also had "feebleness in walking," complained of intense neuralgic pains in his neck, which extended the whole length of back. Coincident with the first symptom, he had double vision, and this interfered considerably with locomotion. At the present time there is pronounced difficulty in walking, a stammering in speech, an incöordination in arms. Complains continually of numbness in entire left side, is quite irritable and occasionally "talks at random on various subjects."

Case 12. - Brother of preceding. Age seventy-two. Is exhibiting prodromata of the disease. He complains of extreme nervousness, insomnia and vertigo; has trouble in walking, but this is only transient, and is only prominent when he is excited. He dates these symptoms from the age of sixty-eight.

Information concerning cases $1,2,4,5,6,7$, and 8 were gained entirely by correspondence. Description of cases $3,9,11$, and 12 were obtained by personal inquiry. The relative giving information concerning these states: "They are all taken at the age of 
sixty in the lower limbs, their gait is unsteady, then it creeps up gradually to the arms, and finally the speech is affected. It does not affect their seeing or hearing, and their mental faculties remain bright."

Case 10.- Mrs. C. Age seventy-five. Admitted to the Michigan Asylum for the Insane, July 18, 1893.

Previous History. - Physical health has been poor for the last ten years, but there appeared to be no localized physical trouble. She complained of various visceral pains and there was habitual constipation.

At the age of fifty-five it was noticed that there was an inability to control her muscles in walking, and this gradually increased until one year afterward; she was then unable to walk in a straight line and required assistance. At this time she complained of prins in back and legs, but these were not severe. This condition continued until the age of fifty-eight, when incöordination of muscles of arms and fingers developed. Six months after this, speech was noticed to be stammering, and she would hesitate before speaking. In the course of a year she became entirely helpless, and difficulty in walking, incorordination in arms, and trouble in articulation became exaggerated. No mental symptoms were noted at that time and no new symptoms developed. At the age of seventy-three she would occasionally become confused, irritable, and had delusions concerning her surroundings. These were persecutory in character and referred particularly to her relatives. This condition would be alternated with one of depression, during which she would become quite emotional, but developed no depressive delusion.

Repeated examinations have been made during her residence in the asylum.

July 20, 1893.-Head small, but symmetrical. Marked tremor in facial muscles in masticating and also in speaking. Muscularity well preserved. Pupils equal and normal, and react. No extra or intra-ocular trouble noticed.

Speech.-Markedly ataxic and articulation slow. Explosive quality well marked. Does not misplace words. Deglutition is performed without difficulty.

Arms.-There is marked tremor, volitional in character, and also on voluntary movement extreme ataxia is developed. Muscular sense is slightly involved; in attempting to pick up small objects and in buttoning clothes this is exaggerated. Terminal phalanx of thumb is flexed, but no other deformity is noticed. No atrophy or Vor. LI - No. III - F 
spasm, and no involvement of sensation. Muscular power is good; ataxia is bi-lateral and apparently equal.

Legs.-Pronounced ataxia in walking; unable to walk unsupported. On closing eyes, this is increased and there is a characteristic swaying movement of the whole body. On several occasions she fell and was unable to regain ber feet. In sitting or in recumbent posture there is marked tremor of upper portion of body, increased on volition. No atrophy or spasm, and muscular sense is normal, although slight dulling to touch is noticed on inner sides of both legs extending from knee to ankle. This, however, could not be accurately localized or determined. Sensation is delayed, probably dependent on mental condition. No deformity. Emotion always caused an exaggeration of her symptoms.

Reflexes.-Knee jerks active, slight right ankle clonus, no patellar clonus. Knee jerks transferred. Reinforcement has no obvious effect. Muscular and superficial reflexes active all over body.

Psychical Examination.-Slight irritability, mentalization is slow, but she displays a full realization of her surroundings, converses quite rationally on many subjects, and conversation is coherent. No delusion or sensory perversion noted. Evidently a condition of terminial dementia.

Electrical Examination.-There is increased faradic excitability in muscles of face, arms and legs; otherwise reactions are normal.

September 5, 1893.- Intermittent twitches of both eye-balls can be noted on fixation, although this is not constant. Articulation is markedly ataxic and explosive. Difficulty in walking has increased, and she now requires assistance at all times.

September 14, 1893.-To-day she had a vertiginous attack. She fell, but apparently did not lose consciousness. Afterward complained of headache, which gradually subsided. There is a degree of arterio-sclerosis present.

January 20, 1894.- Pupils are somewhat contracted, react normally to accommodation, but sluggishly to light. Tremor of facial muscles is now pronounced. Tongue is protruded straight, but after a time there is quite a noticeable coarse tremor. Nails on hands are more curved and more concave in shape, but present no decided trophic change.

April 1, 1894. - Examination of eyes (Dr. Oliver A. La Crone). Pupils are slightly contracted. React normally to accommoda- 
tion and light. No extra or intra-ocular paralysis. R. E. small striæ throughout center of lens. Optic disc shows beginning atrophy with limitation of visual field. Vision $\frac{20}{100}$, with $+3.50 \mathrm{D}$; can read No. 24 Snellen at sixteen inches. L. E. lens has a small line through center, retina and optic disc normal, field of vision limited. Vision $\frac{8}{7} 8$, with $+3.50 \mathrm{D}$; can read No. 20 Snellen at sixteen inches.

June 4, 1894.-The symptoms are exaggerated. There is a course tremor in tongue, and speech is pronouncedly ataxic. Psychical condition is unchanged. Atrophy of optic nerve increased.

At the present date, November 27th, there is an increased degree of ataxia in arms and legs. Reflexes are not so active as formerly, but are present in entirety. Other symptoms as before noted.

Case 13.- Sister of patient above described. Age, sixty-seven.

Previous History.-Well, physically and mentally, until two years ago, when she had a severe attack of "influenza." Shortly afterward insomnia developed. Coincident with this, she had trouble with her eyesight, and says at this time she began to see double. Thinks that both eyes were so affected. She was subject to frequent attacks of sudden weakness, described as "giving way of the knees," but does not believe she had vertigo, or at any time lost consciousness. This condition continued until one year ago, when she had considerable difficulty in articulation, and noticed that the letters " $d$ " and " $s$," and words containing them, were pronounced with much difficulty. She would also frequently misplace words, but this could be corrected if she used considerable control and hesitated before speaking. At this time she noticed that she had difficulty in walking; in any sudden movement she would stagger considerably, and in endeavoring to walk along the street she would go "first to one side and then to the other." While walking, she thought that she frequently was on the opposite side, and would misplace houses and persons. The diplopia, difficulty in speaking and in locomotion were always increased by emotion. Habitual constipation had been present for some time, and during the fall of 1893 she had an attack of facial paralysis, evidently of the peripheral form. This entirely disappeared in two months, and since that time she has had no recurrence of the trouble.

Present History.-She states that at the present time all the symptoms enumerated above are present, and in walking she frequently becomes confused, and for a time has no recognition of 
her surroundings. On these occasions her diplopia is worse, and she believes that these attacks are dependent on this. Occasionally she has pains in lower portion of the back, but no history of headache or girdle pain can be elicited. Has never suffered any acute pain, and general physical condition is fairly well maintained, although she is subject to bilious attacks.

Examination March 19, 1894. No tenderness of the scalp, no asymmetry of head, and no tremor of facial or cervical muscles.

Examination of Eyes.-(Dr. Oliver A. LaCrone). Vision R. E. $\frac{50}{50}$ with $+1.50 \mathrm{D}$ equals $\frac{20}{20}$. L. E. $\frac{1}{70}$ with $+1.75 \mathrm{D}$ equals $\frac{8}{2}$. Esophoria which was corrected by $4^{\circ}$ prism. Pupils equal, slightly dilated, react normally. Retinæ normal.

Facial muscles were normal and, although there was slight tremor in articulation, this was not constant. The letters "l," " $d$," and " $t$ " were pronounced with difficulty; words containing them were slurred to some extent, and in talking rapidly explosive and ataxic quality of speech was quite marked. No misplacement of words, and speech was correct in form and arrangement. Tongue protruded straight. No tremor or incöordination. Hearing, as tested by watch, was imperfect in both ears. Bone conduction normal. The senses of taste and smell not involved.

Arms.-Movements could be executed with rapidity. No incöordination, tremor, spasm, or atrophy. No involvement of sensation.

Legs.-In walking there is a distinct halting movement, more prominent on sudden exertion or turning around. In endeavoring to walk a straight line, she invariably swerves from side to side, but had considerable control over these movements. On closing or raising eyes from feet, this difficulty in walking is increased. No loss in muscular power can be detected. There is no spasm or tremor, but at rest slight incöordination is present in legs when executing simple movements. When in sitting posture there is occasional swaying movement of trunk, but she has perfect control over this. No sensory complication.

Reflexes.-Knee jerks on both sides active. Reinforcement has no obvious effect. No patella or ankle clonus. Superficial reflexes normal.

Examination of thoracic and abdominal viscern negative. There is no curvature of spine and no deformity.

Electrical Examination. - Faradic contractility of muscles of arms and legs increased. No other change noted. 
May 8, 1894.-Patient reports herself as being improved. The glasses have aided her considerably in walking and no increase can be noticed in ataxia. No other symptoms bave developed.

Examination of Eyes.-(Dr. Oliver A. LaCrone). Left eye shows beginning optic atrophy at upper part of disc. Otherwise the condition is the same as at last note.

June 21, 1894. - (By correspondence.) The symptoms are reported as being exaggerated. The ataxia has developed in arms, but this is slight, although quite decided. Difficulty in walking is increased, and she now complains of considerable pain in lower portion of back. This, however, is more prominent on exertion. Double vision is increased and glasses do not seem to be effective. There is a mild state of mental depression and she is quite emotional.

During July and August she became quite confused, absentminded, and developed pessimistic delusions. This condition has at present entirely subsided. Her difficulty in walking is increased and difficulty in articulation is more pronounced. The ataxia in arms has not extended since last note.

The following clinical summary can be made:

1. Occurrence of a form of ataxia in thirteen persons in four generations of one family, with a distinct hereditary history.

2. Marked similarity of symptoms in those affected.

3. The onset in all cases noted between the ages of fifty and sixty-five, excepting cases 8 and 12, the first symptom in the former being noted at the age of seventy-two, the initial symptoms appearing in the latter case at the age of sixty-eight.

4. The occurrence of insanity in four of the thirteen cases, namely: Cases 4, 11, 10, and 13. In all cases the form of insanity was undoubtedly dementia, differing in degree and corresponding to a dementia resulting from organic brain disease. 